\title{
Overcoming negative predictions of microRNA expressions to gemcitabine response with FOLFIRINOX in advanced pancreatic cancer patients
}

\author{
Konstantin Schlick*,1,2, Florian Hohla ${ }^{1}$, Frank Hamacher ${ }^{2}$, Hubert Hackl ${ }^{4}$, Clemens \\ Hufnagl 1,2, Steiner Markus 1,2,3, Teresa Magnes ${ }^{1,2}$, Simon Peter Gampenrieder 1,2,3, Thomas \\ Melchardt ${ }^{1,2,3}$, Stefan Stättner ${ }^{5,6}$, Cornelia Hauser-Kronberger ${ }^{7}$, Richard Greil ${ }^{1,2,3}$ \& Gabriel \\ Rinnerthaler $1,2,3$ \\ ${ }^{1}$ IIIrd Medical Department with Hematology \& Medical Oncology, Hemostaseology, Rheumatology \& Infectious Diseases, Oncologic \\ Center, Paracelsus Medical University Salzburg, Müllner Hauptstrasse 48, Salzburg 5020, Austria \\ ${ }^{2}$ Salzburg Cancer Research Institute with Laboratory of Immunological \& Molecular Cancer Research \& Center for Clinical Cancer \\ \& Immunology Trials, Salzburg, Austria \\ ${ }^{3}$ Cancer Cluster Salzburg, Salzburg, Austria \\ ${ }^{4}$ Division of Bioinformatics, Biocenter, Medical University of Innsbruck, Innsbruck, Austria \\ ${ }^{5}$ Department of Surgery, Salzkammergutklinikum, Standort Vöcklabruck, Oberösterreich, Austria \\ ${ }^{6}$ Department of Surgery, Paracelsus Medical University Salzburg, Salzburg, Austria \\ ${ }^{7}$ Department of Pathology, Paracelsus Medical University Salzburg, Salzburg, Austria \\ *Author for correspondence: Tel.: +43 6624482 58868; K.Schlick@salk.at
}

FOLFIRINOX is superior to gemcitabine in patients with pancreatic cancer, but this regimen is associated with toxicity and biomarkers for response are warranted. MicroRNAs can mediate drug resistance and could provide predictive information. Altered expressions of several microRNAs including miR-21$5 p$, miR-10b-5p and miR-34a-5p have been previously linked to a worse response to gemcitabine. We investigated the influence of expression levels in tumor tissue of those three microRNAs on outcome to FOLFIRINOX. Twenty-nine patients with sufficient formalin-fixed paraffin-embedded tumor tissue were identified. There was no significant association between high and low expression groups for these three microRNA. We conclude that polychemotherapy combination can overcome intrinsic negative prognostic factors.

Lay abstract: Treatment outcome with the polychemotherapy FOLFIRINOX is superior to gemcitabine in patients with pancreatic cancer, but FOLFIRINOX can cause severe toxicities. Therefore, we investigated biomarkers that may guide treatment decision. Altered expression of certain microRNAs, small noncoding RNAs, are associated with a worse response to gemcitabine. We found that the expression of the microRNAs miR-21-5p, miR-10b-5p and miR-34a-5p have no preditive value in patients treated with FOLFIRINOX. We therefore, conclude that polychemotherapy combination can overcome intrinsic negative prognostic factors.

First draft submitted: 15 July 2020; Accepted for publication: 18 September 2020; Published online:

1 December 2020

Keywords: FOLFIRINOX • gemcitabine • miRNA • pancreatic cancer

Pancreatic cancer (PC) is the third leading cause of cancer-related death in men and women in the USA and is anticipated to take the second place in 2020 ranking second after lung cancer [1]. In 2019, 45,750 PC deaths and 56,770 new cases were estimated in the USA alone; however, by 2030, 63,000 expected cancer death from PC are predicted, making it the number one cause of cancer-related death in the next 10 years [2]. It is estimated that, by 2040, the total number of cases in the EU will increase by more than $30 \%$ [3]. 
PC remains one of the most devastating malignant diseases and outcome is dismal with a 5-year survival rate of only $5 \%$ [4]. A curative treatment approach with a radical surgical resection can only be offered to less than $20 \%$ of cases as most patients are either in an advanced stage of disease or present with incurable metastatic disease.

FOLFIRINOX (5-fluorouracil/leucovorin, irinotecan and oxaliplatin) is a highly active chemotherapy regimen in the adjuvant setting [5] and for the first-line treatment of patients with unresectable advanced or metastatic pancreatic cancer (APC) [6]. In a pivotal adjuvant trial, a statistically significant overall survival (OS) benefit was demonstrated for modified-FOLFIRINOX compared with gemcitabine in PC patients with an increase of 19.4 months (54.4 vs 35.0 months, stratified hazard ratio [HR] for death $0.64 ; 95 \%$ CI: $0.48-0.86 ; \mathrm{p}=0.003$ ) [5]. Additionally, gemcitabine plus capecitabine led to a significant increase of OS from 25.5 to 28.0 months (HR: 0.82; 95\% CI: 0.68-0.98; $\mathrm{p}=0.032)$ compared with gemcitabine mono treatment in the adjuvant setting [7].

In patients with APC, FOLFIRINOX showed a significantly longer OS of 11.1 versus 6.8 months (HR: 0.57; $\mathrm{p}$ $<0.001$ ) and a significant longer progression-free survival (PFS) of 6.4 versus 3.3 months (HR: $0.47 ; \mathrm{p}<0.001$ ) compared with the former treatment standard gemcitabine in a Phase III trial including 342 patients [6]. However, this advantage in survival comes at the cost of toxicity with a significant increase of grade 3 or 4 adverse events. A dual chemotherapy combination of gemcitabine plus nab-paclitaxel was also more effective compared with gemcitabine alone as first-line treatment in APC with a significant increase of OS from 6.7 to 8.5 months (HR: 0.72; p < 0.001) and PFS from 3.7 to 5.5 (HR: 0.69; p < 0.001), respectively [7]. Based on the results of those two trials, the European Society of Medical Oncology and the American Society of Clinical Oncology recommend FOLFIRINOX or a gemcitabine plus nab-paclitaxel combination as first-line treatment in fit patients with APC $[8,9]$.

MicroRNAs are currently under investigation in various cancer entities for their diagnostic, prognostic and predictive role as biomarkers [10]. MicroRNAs are small, approximately 22 nucleotides long noncoding singlestranded RNAs, regulating gene expression at a post-transcriptional level. The human genome may encode more than 1000 microRNAs and approximately $60 \%$ of human genes are regulated by microRNAs. Besides other functions, they are known to be involved in tumor evolution [11] including regulation of angiogenesis [12] and development of treatment resistance [13]. However, no existing microRNA panel is currently endorsed for clinical use in therapy response. Altered microRNA expression profiles were described in different malignancies [14]. Generally, microRNAs act as tumor suppressors, by negatively regulating oncogenes, genes that promote cell proliferation, as well as genes that inhibit cell division $[15,16]$. Therefore, dysregulation can lead to carcinogenesis with loss of normal function resulting in altered expression of, for example, tumor suppressor genes. Based on these findings, it is not surprising that microRNAs can be used for tumor diagnosis and can provide both prognostic and predictive information in different cancer entities.

MicroRNA can be analyzed from several body fluids like blood, but also from tissue specimen. Although microRNA analysis from blood samples has the advantage of being noninvasive and samples are easily accessible with a minimum risk for the patient, the measured microRNAs are not cancer and disease specific [17-19]. In contrast, microRNA analyses from tumor tissue specimen allow better conclusions to be drawn about tumorassociated expressions. Additionally, archived tumor tissue is commonly available compared with archived blood samples, which are rare. Therefore, unplanned retrospective analysis is more feasible from tumor tissue.

In PC, a distinct microRNA expression profile compared with benign lesions has been observed [20-24]. Using microRNA expression signatures consisting of 20 to 32 microRNAs, a clear discrimination between healthy, inflamed and cancerous pancreatic tissue can be made $[20,22,24]$. These findings are of clinical relevance, because histological clarification of pancreatic lesions is often challenging, particularly in tissues obtained by endo-sonography-assisted fine-needle biopsies. Furthermore, plasma levels of circulating miR-221 are significantly higher in samples from patients with PC compared with healthy controls and the expression level is correlated with prognosis [25].

Beyond the diagnostic value of microRNA expression profiles, several microRNAs like miR-155, miR-21, let7, miR200c, miR34a have shown to be prognostic and predictive [20,26-35].

The predictive value of microRNAs for therapy response under gemcitabine in PC patients was shown in vitro and in vivo. An altered expression of miR-21 (overexpression), miR-10b and miR-34a (downregulation) has been previously associated with worse survival under gemcitabine chemotherapy [29-31,36,37]. The aim of this study was to investigate the influence of those three microRNAs on the treatment outcome of FOLFIRINOX in patients with APC and whether FOLFIRINOX can overcome these negative predictors to gemcitabine treatment. 


\section{Materials \& methods}

\section{Study population}

In this single center study at the IIIrd Medical Department of the Paracelsus Medical University Salzburg, consecutive patients with advanced PC eligible for treatment with FOLFIRINOX between 2010 and 2012 were retrospectively analyzed. All patients treated with at least one dose of FOLFIRINOX as first-line treatment, an age over 18 years and sufficient available tumor tissue for microRNA analysis were eligible for our analyses.

Dose modifications were made at the discretion of the treating physician. As per clinical standard, performance status and comorbidities where considered for primary dose modifications. Furthermore, treatment-associated toxicities resulted in secondary dose modifications as decided by the investigator. As per our institutional standard, treatment was continued until disease progression or documentation of unacceptable toxicities. We retrospectively evaluated patient characteristics, Eastern Cooperative Oncology Group (ECOG) performance score [38], date of diagnosis, start of FOLFIRINOX treatment, dose modifications during treatment, toxicity, response rates, PFS and OS based on review of patient's medical records and radiology reports. Tumor response was defined by using CT scans classified into partial response, stable disease or progressive disease according to WHO criteria taking tumor marker response into account [39].

\section{Tissue samples}

Formalin-fixed paraffin-embedded (FFPE) tissue samples from primary tumors, or if available, from metastatic sites, were selected by an experienced pathologist (C Hauser-Kronberger). All tissue samples were collected prior to the start of first-line chemotherapy for metastatic disease. Three to five $10-\mu \mathrm{m}$ paraffin-sections were cut from each block without micro- or macro-dissection and placed in sterile Eppendorf tubes.

\section{RNA purification \& microRNA expression analysis}

Total RNA was purified from FFPE-Tissue using the mirVana ${ }^{T M}$ microRNA Isolation Kit from Ambion ${ }^{\circledR}$. The concentration and purity of total RNA was determined by measuring $1.5 \mu \mathrm{l}$ from RNA isolation with NanoDrop (ThermoFisher Scientific, MA, USA) and $1 \mu \mathrm{g}$ was reversely transcribed to cDNA using the TaqMan ${ }^{\circledR}$ Reverse Transcriptase Kit and specific reverse PCR primers (TaqMan MicroRNA Assay) according to the manufacturer's instructions. (For microRNA target sequence see Supplementary Table 2) MicroRNA expression was quantified using microRNA-specific forward primers (TaqMan MicroRNA Assay) for hsa-miR-21-5p, hsa-miR-10b-5p, hsamiR-34a-5p and RNU6b in combination with the TaqMan Universal PCR Master Mix by quantitative real-time PCR.

\section{Statistical analysis}

PFS was defined as the time from start of FOLFIRINOX treatment until date of confirmed progression or death from any cause. OS was as defined from start of FOLFIRINOX treatment until death from any cause. Data analysis for this retrospective study was descriptive in nature and presented in medians and ranges (95\% CI). PFS and OS estimates were obtained using the Kaplan-Meier (KM) method. The median follow-up duration was measured by reverse KM estimator. Comparing survival curves by the use of Cox regression univariate analyses including HR with 95\% CI. The HR describes the relative risk of the occurrence of an end point of interest based on comparison of event rates between patient groups. The HR is equivalent to the odds that an individual in the group with the higher hazard reaches the end point first. The association of microRNA expression, dichotomized based on median expression, with PFS or OS was analyzed for each microRNA using a log rank test.

Comparisons of microRNA expressions between adjuvant gemcitabine treatment groups were performed using the Wilcoxon rank sum exact test.

All statistical analyses were performed using the statistical software environment $R$ (packages survival). A p-value of $<0.05$ was considered to be statistically significant with regard to median OS with $95 \%$ CI.

\section{Ethics}

This analysis was approved by the Ethics Committee of the federal state of Salzburg, Austria (IRB number: 415$\mathrm{EP} / 73 / 236-2013)$. Due to the retrospective character, the pseudonymized analysis and the death of all patients at the time point of data analysis, the committee waived the requirement for informed consent. 


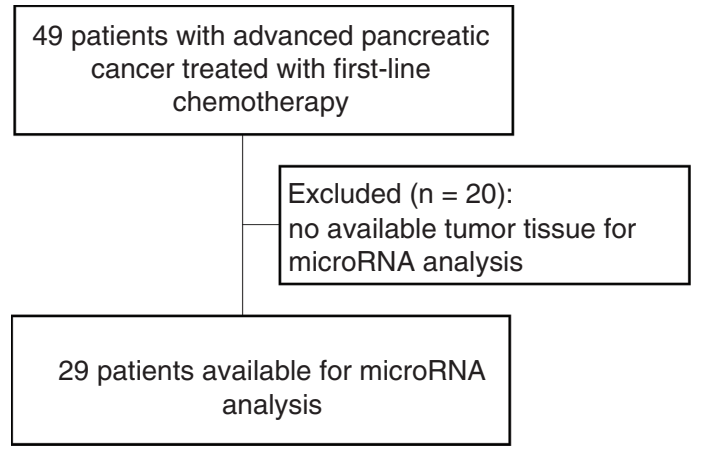

Figure 1. Consort diagram.

\begin{tabular}{l} 
Table 1. Patient characteristics with available tumor tissue. \\
\begin{tabular}{|l|l|}
\hline Characteristic & $\mathrm{n}=29$ \\
\hline Age & 63 \\
\hline - Median & $45-75$ \\
\hline- Range & \\
\hline Gender & $15(52 \%)$ \\
\hline - Female & $14(48 \%)$ \\
\hline - Male & \\
\hline Grading & $20(69 \%)$ \\
\hline - G2 & $8(28 \%)$ \\
\hline - G3 & $1(3 \%)$ \\
\hline - Unknown & \\
\hline Histology & $28(97 \%)$ \\
\hline - Adenocarcinoma & $1(3 \%)$ \\
\hline - Adenosquamous carcinoma & \\
\hline Gemcitabine treatment & $8(28 \%)$ \\
\hline - Adjuvant & $11(38 \%)$ \\
\hline - After progression on FOLFIRINOX \\
\hline Relapse or primary advanced & $3(10 \%)$ \\
\hline - Primary locally advanced non-metastatic & $15(52 \%)$ \\
\hline - Primary metastatic & $11(38 \%)$ \\
\hline - Unresectable relapse after initial resection & \\
\hline
\end{tabular} \\
\hline
\end{tabular}

\section{Results}

Forty-nine patients with APC treated between 2010 and 2012 at the IIIrd Medical Department of the Paracelsus Medical University Salzburg with first-line FOLFIRINOX were identified. In twenty-nine patients (59\% of the study cohort), sufficient FFPE tumor tissue was available for our analyses (Figure 1). Median age at diagnosis was 63 years (45-75 years), 14 (48\%) were male and the majority of our female and male study cohort had an ECOG performance status of 0 or 1 as expected for a selection for polychemotherapy. Patient's baseline characteristics are outlined in Table 1. Forty-two samples derived from primary tumor and 16 (28\%) from metastasis (3 lymph node, 3 liver, 2 lung, 1 pleural, 2 soft tissue, 3 skin, 1 ovary, 1 bone marrow). Twenty-two (38\%) specimens were achieved by core biopsy and $36(62 \%)$ by surgery.

Overall median PFS (95\% CI) and median OS (95\% CI) for the whole study cohort was 4.67 (3.48-7.73) and 10.52 (9.66-13.12) months, respectively.

Upon purification of RNA, median RNA concentration was $0.09 \mu \mathrm{g} / \mu \mathrm{l}(0.02-0.83)$ with median sample purity of 1.65 (1.10-2.87; 260/280 $\mathrm{nm}$ ratio) and 1.17 (0.07-6.34; 250/230 nm ratio).

Patients were divided into a low $(\mathrm{n}=14)$ and high $(\mathrm{n}=15)$ expression group, based on the median expression level of the microRNA in the tumor. MicroRNA expression levels are listed in Supplementary Table 1. There was no significant association between high and low expression groups with miR-21-5p $(\mathrm{HR}=1.1 ; \mathrm{p}=0.78 ; \mathrm{HR}=1.12 ; \mathrm{p}$ $=0.76)$, miR-10b-5p $(\mathrm{HR}=0.67 ; \mathrm{p}=0.26 ; \mathrm{HR}=0.71 ; \mathrm{p}=0.34)$ and $\operatorname{miR}-34 \mathrm{a}-5 \mathrm{p}(\mathrm{HR}=0.89 ; \mathrm{p}=0.75$; 

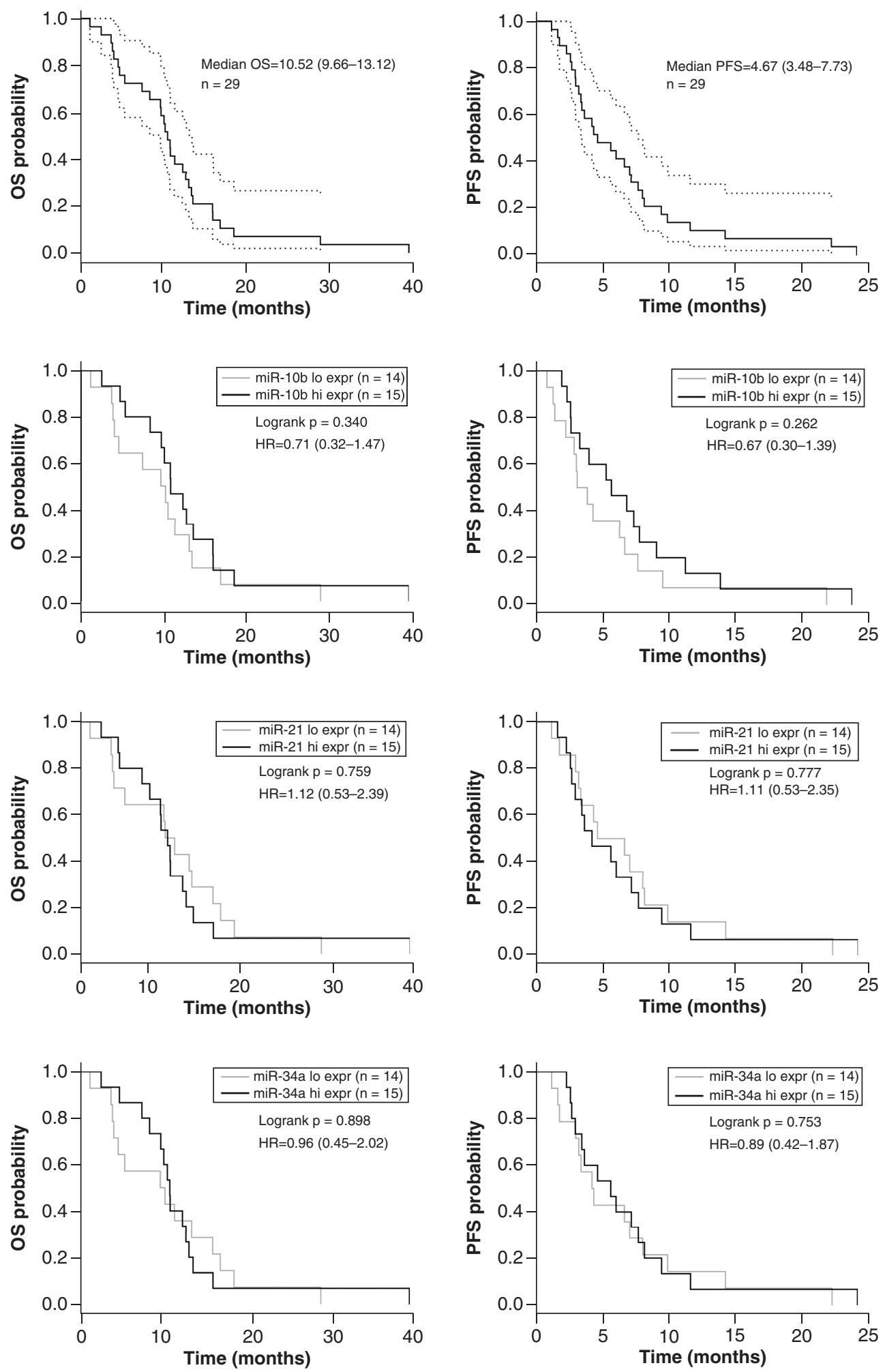

Figure 2. Overall and progression-free survival Kaplan-Meier plots for the overall population and for the microRNA expression groups (high vs low) of miR-21-5p, miR-10b-5p and miR-34a-5p. Median OS and median PFS are shown for overall study population including $\mathrm{Cls}$ in the first row. In the following blots median OS and median PFS based on the median dichotomized microRNA expression groups (high vs low) are illustrated for miR-21-5p, miR-10b-5p and miR-34a-5p.

Hi expr: High expression; HR: Hazard ratio; Low expr: Low expression; OS: Overall survival; PFS: Progression-free survival. 
$\mathrm{HR}=0.96 ; \mathrm{p}=0.90$ ), with regard to PFS and OS, respectively (see Figure 2 for KM plots \& Supplementary Figure 1 for correlation analyses). No statistically significant differences of the expressions of miR-21 ( $\mathrm{p}=0.55)$, miR-10p $(\mathrm{p}=0.37)$ and miR-34a $(\mathrm{p}=0.79)$, were seen between patients treated with adjuvant gemcitabine compared with patients with no adjuvant gemcitabine therapy.

\section{Discussion}

To our knowledge, this is the first study to demonstrate that FOLFIRINOX polychemotherapy can overcome the poor predictive value of certain microRNAs shown in APC patients treated with gemcitabine. With higher response rates and lower HRs for recurrence and death, FOLFIRINOX seems to be more effective but also more toxic than the gemcitabine combination. Grade 3-4 side effects occur quite frequently, which raises questions of patient selection and leads to dose-reduction and dose-modified schedules. Proper patient selection is crucial to identify those that are most likely to benefit from aggressive chemotherapy approaches and separate those, who will likely have only little benefit due to increased rates of severe side effects. However, no prospectively validated models are available to guide decision making for an upfront patient selection.

MiR-21 is commonly considered an oncogene and exhibits anti-apoptotic activity. It enhances cellular proliferation, migration and invasion, leading to aggressive tumor progression. In vitro studies suggested that miR-21 conferred chemoresistance via modulation of apoptosis, Akt phosphorylation and expression of genes involved in cellular invasiveness [17]. Further miR-21 confers chemoresistance by targeting FAs-ligand and inhibiting gemcitabineinduced apoptosis. Furthermore miR-21 induces 5-fluorouacil resistance in human pancreatic cells by regulating PTEN and PDCD4 published by Wei et al. [40]. Hwang et al. showed a negative impact of miR-21 in PC patients treated with Gem or 5-FU containing adjuvant regimen [30]. Therefore, an altered expression of miR-21 (overexpression), miR-10b and miR-34a (downregulation) has been previously associated with worse survival under gemcitabine chemotherapy [29-31,36,37].

In our analyses, no influence by the expression levels of miR-21-5p-, miR-10b-5p and miR-34a-5p on PFS and OS could be detected. This suggests that these mRNAs, in contrast to gemcitabine, do not play a role for FOLFIRINOX efficacy. With polychemotherapy, one might overcome negative prediction of certain mRNA to single agent therapy.

Despite the investigation of miRNA in tumor tissue, expression profiles of circulation miRNAs have also been investigated as potential biomarkers for PC. Meijer $e$ t al. demonstrated that a decline in plasma miR-181a-5p levels after 5-6 cycles of FOLFIRINOX was associated with better prognosis and may be useful for guiding therapeutic choices and surgical exploration [41]. This association was not observed in a second cohort of patients treated with gemcitabine plus nab-paclitaxel. In vitro analyses detected an increased sensitivity of PC cells lines to oxaliplatin when miR-181a-5p was inhibited.

Several limitations should be considered in the present study. First, the rather small sample size precludes a definitive conclusion about the predictive and prognostic value of the investigated miRNAs. Furthermore, the lack of a control group not treated with FOLFIRINOX is a further limitation. However, our study does not provide any evidence that the three investigated micro-RNAs do have any influence on the efficacy of FOLFIRINOX as first-line therapy of APC.

\section{Future perspective}

Expression levels of miR-21-5p, miR-10b-5p and miR-34a-5p in pancreatic tumor tissue do not predict treatment outcome to FOLFIRINOX. As altered expressions of these microRNAs were shown to be associated with a worse response to gemcitabine, these markers may help to select patients for a treatment with FOLFIRINOX or a gemcitabine-based regimen, respectively. Hopefully in the near future more biomarkers will arise and be validated for novel therapeutic agents like nab-Paclitaxel or nano-liposomal-irinotecan in order to properly select patients to certain personalized chemotherapeutic regiment.

\section{Author contributions}

$\mathrm{G}$ Rinnerthaler and $\mathrm{H}$ Hackl conceived and designed the experiments. C Hauser-Kronberger selected the tumor tissues. M Steiner and C Hufnagl performed the experiments. K Schlick, F Hamacher, T Magnes, SP Gampenrieder, T Melchardt, S Stättner and G Rinnerthaler collected the clinical data. K Schlick, H Hackl, M Steiner and G Rinnerthaler analyzed the data and drafted the manuscript. R Greil and SP Gampenrieder assisted in preparing the manuscript. All authors critically revised the manuscript and approved the final manuscript. 
Financial \& competing interests disclosure

This research was funded by the Reiner-Brettenthaler grant 2013 from the Medical Association of Salzburg. The authors have no other relevant affiliations or financial involvement with any organization or entity with a financial interest in or financial conflict with the subject matter or materials discussed in the manuscript apart from those disclosed.

No writing assistance was utilized in the production of this manuscript.

Ethical conduct of research

The authors state that they have obtained appropriate institutional review board approval or have followed the principles outlined in the Declaration of Helsinki for all human or animal experimental investigations.

\section{Supplementary data}

To view the supplementary data that accompany this paper please visit the journal website at: www.futurescience.com/doi/suppl/10.2144/fsoa-2020-0128

\section{Open access}

This work is licensed under the Creative Commons Attribution 4.0 License. To view a copy of this license, visit http://creativecomm ons.org/licenses/by/4.0/

\section{Summary points}

- FOLFIRINOX is superior to gemcitabine in patients with pancreatic cancer (PC), but this polychemotherapy regimen is associated with more toxicities.

- Biomarkers guiding treatment decision for PC patients are lacking.

- Tissue expression of several microRNAs like miR-21-5p, miR-10b-5p and miR-34a-5p have been previously linked to worse outcome in gemcitabine treatment patients with PC.

- In this retrospective analysis of patients treated with FOLFIRINOX, miR-21-5p, miR-10b-5p and miR-34a-5p tissue expressions levels were not correlated with outcome.

- We conclude that polychemotherapy combination can overcome intrinsic negative prognostic factors.

\section{References}

1. Miller KD, Nogueira L, Mariotto AB et al. Cancer treatment and survivorship statistics, 2019. CA Cancer J. Clin. 69, 363-385 (2019).

2. Rahib L, Smith BD, Aizenberg R et al. Projecting cancer incidence and deaths to 2030: the unexpected burden of thyroid, liver, and pancreas cancers in the United States. Cancer Res. 74, 2913-2921 (2014).

3. Maisonneuve P. Epidemiology and burden of pancreatic cancer. Presse Med. 48, e113-e123 (2019).

4. Niederhuber JE, Brennan MF, Menck HR. The National Cancer Data Base report on pancreatic cancer. Cancer 76, 1671-1677 (1995).

5. Conroy T, Hammel P, Hebbar M et al. FOLFIRINOX or gemcitabine as adjuvant therapy for pancreatic cancer. N. Engl. J. Med. 379, 2395-2406 (2018).

6. Conroy T, Desseigne F, Ychou M et al. FOLFIRINOX versus gemcitabine for metastatic pancreatic cancer. N. Engl. J. Med. 364, 1817-1825 (2011).

7. Von Hoff DD, Ervin T, Arena FP et al. Increased survival in pancreatic cancer with nab-paclitaxel plus gemcitabine. N. Engl. J. Med. 369, 1691-1703 (2013).

8. Ducreux M, Cuhna AS, Caramella C et al. Cancer of the pancreas: ESMO Clinical Practice Guidelines for diagnosis, treatment and follow-up. Ann. Oncol. 26(Suppl. 5), v56-68 (2015).

9. Khorana AA, Mangu PB, Berlin J et al. Potentially curable pancreatic cancer: American Society of Clinical Oncology Clinical Practice Guideline Update. J. Clin. Oncol. DOI: 10.1200/JCO.2017.72.4948 (2017) (Epub ahead of print).

10. Biancur DE, Kimmelman AC. The plasticity of pancreatic cancer metabolism in tumor progression and therapeutic resistance. Biochim. Biophys. Acta Rev. Cancer 1870, 67-75 (2018).

11. Esquela-Kerscher A, Slack FJ. Oncomirs - microRNAs with a role in cancer. Nat. Rev. Cancer 6, 259-269 (2006).

12. Chang SH, Hla T. Gene regulation by RNA binding proteins and microRNAs in angiogenesis. Trends Mol. Med. 17, 650-658 (2011).

13. Liang Z, Wu H, Xia J et al. Involvement of miR-326 in chemotherapy resistance of breast cancer through modulating expression of multidrug resistance-associated protein 1. Biochem. Pharmacol. 79, 817-824 (2010).

14. Calin GA, Croce CM. MicroRNA signatures in human cancers. Nat. Rev. Cancer 6, 857-866 (2006).

15. Calin GA, Croce CM. MicroRNA-cancer connection: the beginning of a new tale. Cancer Res. 66, 7390-7394 (2006). 
16. Serpico D, Molino L, Di Cosimo S. MicroRNAs in breast cancer development and treatment. Cancer Treat. Rev. 40, 595-604 (2014).

17. Wang P, Zhuang L, Zhang J et al. The serum miR-21 level serves as a predictor for the chemosensitivity of advanced pancreatic cancer, and miR-21 expression confers chemoresistance by targeting FasL. Mol. Oncol. 7, 334-345 (2013).

18. Roy S, Benz F, Luedde $\mathrm{T}$ et al. The role of miRNAs in the regulation of inflammatory processes during hepatofibrogenesis. Hepatobiliary Surg. Nutr. 4, 24-33 (2015).

19. Nejad C, Stunden HJ, Gantier MP. A guide to miRNAs in inflammation and innate immune responses. Febs J. 285, 3695-3716 (2018).

20. Bloomston M, Frankel WL, Petrocca F et al. MicroRNA expression patterns to differentiate pancreatic adenocarcinoma from normal pancreas and chronic pancreatitis. JAMA 297, 1901-1908 (2007).

21. Ikenaga N, Ohuchida K, Mizumoto K et al. MicroRNA-203 expression as a new prognostic marker of pancreatic adenocarcinoma. Ann. Surg. Oncol. 17, 3120-3128 (2010).

22. Szafranska AE, Davison TS, John J et al. MicroRNA expression alterations are linked to tumorigenesis and non-neoplastic processes in pancreatic ductal adenocarcinoma. Oncogene 26, 4442-4452 (2007).

23. Szafranska AE, Doleshal M, Edmunds HS et al. Analysis of microRNAs in pancreatic fine-needle aspirates can classify benign and malignant tissues. Clin. Chem. 54, 1716-1724 (2008).

24. Schultz NA, Werner J, Willenbrock $\mathrm{H}$ et al. MicroRNA expression profiles associated with pancreatic adenocarcinoma and ampullary adenocarcinoma. Mod. Pathol. 25, 1609-1622 (2012).

25. Kawaguchi T, Komatsu S, Ichikawa D et al. Clinical impact of circulating miR-221 in plasma of patients with pancreatic cancer. $B r$. J. Cancer 108, 361-369 (2013).

26. Greither T, Grochola LF, Udelnow A et al. Elevated expression of microRNAs 155, 203, 210 and 222 in pancreatic tumors is associated with poorer survival. Int. J. Cancer 126, 73-80 (2010).

27. Jamieson NB, Morran DC, Morton JP et al. MicroRNA molecular profiles associated with diagnosis, clinicopathologic criteria, and overall survival in patients with resectable pancreatic ductal adenocarcinoma. Clin. Cancer Res. 18, 534-545 (2012).

28. Yu J, Ohuchida K, Mizumoto K et al. MicroRNA, hsa-miR-200c, is an independent prognostic factor in pancreatic cancer and its upregulation inhibits pancreatic cancer invasion but increases cell proliferation. Mol. Cancer 9, 169 (2010).

29. Giovannetti E, Funel N, Peters GJ et al. MicroRNA-21 in pancreatic cancer: correlation with clinical outcome and pharmacologic aspects underlying its role in the modulation of gemcitabine activity. Cancer Res. 70, 4528-4538 (2010).

30. Hwang JH, Voortman J, Giovannetti E et al. Identification of microRNA-21 as a biomarker for chemoresistance and clinical outcome following adjuvant therapy in resectable pancreatic cancer. PLoS ONE 5, e10630 (2010).

31. Preis M, Gardner TB, Gordon SR et al. MicroRNA-10b expression correlates with response to neoadjuvant therapy and survival in pancreatic ductal adenocarcinoma. Clin. Cancer Res. 17, 5812-5821 (2011).

32. Wang $\mathrm{P}$, Chen L, Zhang J et al. Methylation-mediated silencing of the miR-124 genes facilitates pancreatic cancer progression and metastasis by targeting Rac1. Oncogene 33, 514-524 (2014).

33. Tavano F, di Mola FF, Piepoli A et al. Changes in miR-143 and miR-21 expression and clinicopathological correlations in pancreatic cancers. Pancreas 41, 1280-1284 (2012).

34. Caponi S, Funel N, Frampton AE et al. The good, the bad and the ugly: a tale of miR-101, miR-21 and miR-155 in pancreatic intraductal papillary mucinous neoplasms. Ann. Oncol. 24, 734-741 (2013).

35. Papaconstantinou IG, Manta A, Gazouli M et al. Expression of microRNAs in patients with pancreatic cancer and its prognostic significance. Pancreas 42, 67-71 (2013).

36. Park JK, Lee EJ, Esau C et al. Antisense inhibition of microRNA-21 or -221 arrests cell cycle, induces apoptosis, and sensitizes the effects of gemcitabine in pancreatic adenocarcinoma. Pancreas 38, e190-199 (2009).

37. Ji Q, Hao X, Zhang M et al. MicroRNA miR-34 inhibits human pancreatic cancer tumor-initiating cells. PLoS ONE 4, e6816 (2009).

38. Oken MM, Creech RH, Tormey DC et al. Toxicity and response criteria of the Eastern Cooperative Oncology Group. Am. J. Clin. Oncol. 5, 649-55 (1982).

39. Miller AB, Hoogstraten B, Staquet M et al. Reporting results of cancer treatment. Cancer 47, 207-214 (1981).

40. Wei X, Wang W, Wang $\mathrm{L}$ et al. MicroRNA-21 induces 5-fluorouracil resistance in human pancreatic cancer cells by regulating PTEN and PDCD4. Cancer Med. 5, 693-702 (2016).

41. Meijer LL, Garajova I, Caparello C et al. Plasma miR-181a-5p downregulation predicts response and improved survival after FOLFIRINOX in pancreatic ductal adenocarcinoma. Ann. Surg. 271(6), 1137-1147 (2018). 